\title{
Impacto de maloclusiones sobre la calidad de vida y necesidad de tratamiento ortodóntico en escolares de dos escuelas privadas Azogues - Ecuador, 2015
}

\author{
Impact of malocclusions on quality of life and Need for Orthodontic Treatment in schoolchildren of two \\ private schools Azogues - Ecuador, 2015
}

Cristina Crespo 1,a , Cristina Domínguez 1,b, Fernando Vallejo ${ }^{1, c}$, Carlos Liñán ${ }^{2, d}$, César Del Castillo ${ }^{3, e}$, Roberto Antonio León-Manco ${ }^{3, f}$, Alexis Evangelista ${ }^{4, g}$

\section{RESUMEN}

Objetivos: Evaluar el impacto de las condiciones orales atribuidas a maloclusiones sobre la calidad de vida a través del CS Child-OIDP y Necesidad De Tratamiento Ortodóntico a través del INTO en escolares de 11 a 12 años de dos escuelas privadas en Azogues-Ecuador, año 2015. Material y métodos: Esta investigacion evaluó a 170 escolares para determinar el impacto de las maloclusiones sobre la calidad de vida, mediante la versión peruana del Child-OIDP, utilizando únicamente. las condiciones atribuidas a maloclusiones, (CS Child-OIDP Posición de los dientes, espacios, tamaño y forma y deformidades cracenofaciales), sobre los desempeños diarios, y la Necesidad de Tratamiento De Ortodoncia mediante el Indice de Necesidad de Tratamiento de Ortodoncia (INTO) . Resultados: Las condiciones más prevalentes fueron: posición de los dientes (63,5\%), y dientes separados (40,5\%); los desempeños más afectados fueron comer (65,3\%) y sonreír $(51,8 \%)$; respecto de la necesidad de tratamiento ortodóntico según el componente estético, el 91,18\% de los escolares no tuvieron necesidad de tratamiento y según el componente de salud dental, el 10\%; al asociar las dos variables, se encontró diferencia estadísticamente significativa en el desempeño sonreír p=0,02; según el Componente de Salud Dental. Conclusiones: La condición más prevalente fue posición de los dientes, los desempeños diarios más afectados fueron comer y sonreír, al asociar Necesidad de Tratamiento y desempeños afectados en los dos componentes revelaron a los desempeños comer y sonreír como los más afectados, existiendo significancia estadística en el desempeño sonreír.

PALABRAS CLAVE: Calidad de vida, Child-OIDP, necesidad de tratamiento, INTO.

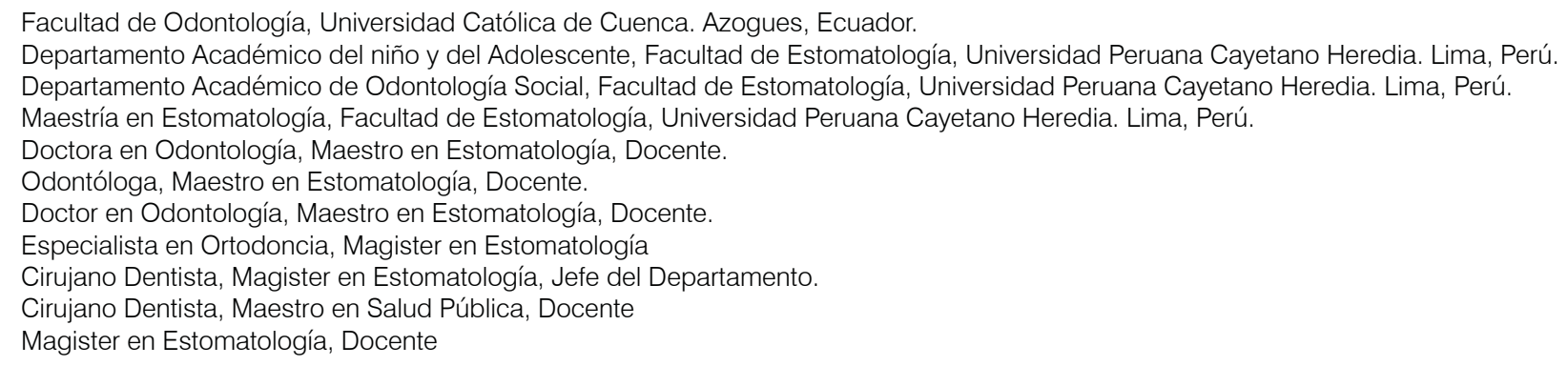




\section{SUMMARY}

Objectives: To evaluate the impact of oral conditions attributed to malocclusions on quality of life through CS Child-OIDP and Need for Orthodontic Treatment through INTO in schoolchildren aged 11 to 12 from two private schools in Azogues-Ecuador, year 2015. Material and Methods: This study evaluated 170 schoolchildren to determine the impact of malocclusions on quality of life, using the Peruvian version of the Child-OIDP, but only using the conditions attributed to malocclusions, (CS Child-OIDP position of teeth, spaces, size And form and cracenofacial deformities), on daily performance, and the need for orthodontic treatment through the need for orthodontic treatment index (INTO). Results: The most prevalent conditions were Position of the teeth (63.5\%), and Separated Teeth (40.5\%); The most affected performances were eating (65.3\%) and smiling (51.8); regarding the need for orthodontic treatment according to the aesthetic component, $91.18 \%$ of the students did not need treatment and according to the Dental Health Component 10\%; when associating the two variables, we found statistically significant difference only in the performance smile $\mathrm{p}=0.02$ according to the Dental Health Component. Conclusions: The most prevalent condition was position of the teeth, the most affected daily performances were eating and smiling, when associating Treatment Need and affected performances in the two components revealed to eating and smiling performance as the most affected, there being statistical significance in performance smile.

\section{KEY WORDS: Quality of life, Child-OIDP, need for treatment, INTO.}

\section{INTRODUCCIÓN}

La interrelación entre la salud bucodental y calidad de vida cada vez adquiere mayor relevancia y despierta en la comunidad científica un interés plasmado en innumerables investigaciones, algunos estudios revelan que anualmente 160 millones de horas de trabajo y 1,57 millones de días de pérdida de clases se deben a desórdenes orales afectando el diario vivir de los individuos sin importar la etapa del ciclo vital en que se encuentren (1).

Según la Organización Mundial de la Salud (OMS) para que un individuo posea una buena calidad de vida, es menester que exista ausencia de dolor orofacial, infecciones, llagas bucales, enfermedades periodontales, caries, pérdida dentaria entre algunos trastornos, pues ello puede afectar significativamente en los desempeños diarios del hogar, escuela, trabajo, juegos, etc. En este sentido la OMS también afirma que las maloclusiones son consideradas la tercera patología más prevalente luego de caries y enfermedad Periodontal, y la Organización Panamericana de la Salud (OPS), manifiesta los altos índices de incidencia y prevalencia de maloclusiones, superando el $80 \%$ de la población; en tal razón son importantes y frecuentes motivos de consulta odontológica $(1,2)$.

Una maloclusión es la desviación de los parámetros normales de la oclusión ideal y exige intervención ortodóntica que solucione los problemas estéticos y funcionales, sin embargo, hay que considerar que existe también un efecto psicosocial y de calidad de vida especialmente durante la niñez y adolescencia (3). Algunos estudios consideran que a medida que los niños se acercan a la adolescencia inician su proceso de integración al entorno, adquieren su identidad y juega un papel trascendente el desarrollo de su autoestima y autopercepción; en tal razón una mal oclusión en cualquier grado es de suponer que afectaría no solo su situación fisiológica, sino su aspecto facial, y una apariencia desagradable influiría negativamente en su personalidad, estableciéndose una triangulo interesante entre maloclusión, aspecto facial y la autoestima de los adolescentes que están en una difícil etapa de transición (4).

Para ese efecto se han desarrollado diversas metodologías con la intención de cuantificar la presencia y gravedad de las maloclusiones, así como también para determinar la relación e impacto de estas sobre la calidad de vida (5). Para la valoración clínica y la autopercepción de las maloclusiones se utiliza el Índice de Necesidad de Tratamiento de Ortodoncia (INTO), el mismo que ha sido ampliamente utilizado para determinar prevalencias en estudios epidemiológicos y paralelamente para establecer la necesidad de tratamiento de Ortodoncia (6). 
Existen índices que están diseñados para medir calidad de vida relacionada a la salud oral en niños como son: Child Perception Questionnaire, Michigan OHRQoL Scale y el Child OIDP (Oral Impacts on Daily Performance); y cuando los estudios pretenden objetivar el impacto de las maloclusiones sobre la calidad de vida, utilizan índices específicos como el (CS Child-OIDP Index), en donde las condiciones orales percibidas como problemas podrían estar atribuidas a maloclusiones $(6,7)$.

En ese contexto el presente estudio tuvo como objetivo determinar el impacto de las condiciones orales atribuidas a maloclusiones sobre calidad de vida y la necesidad de tratamiento ortodóntico en escolares de 11 y 12 años de las escuelas privadas Providencia y Cesar Cordero Moscoso de la ciudad de Azogues Ecuador, en el año 2015.

\section{MATERIAL Y METODOS}

El presente estudio corresponde a un diseño descriptivo y transversal, la población estuvo conformada por 300 escolares de 11 y 12 años de dos escuelas privadas de la ciudad de Azogues-Ecuador, cuya muestra final quedó conformada por 170 individuos; en el estudio se incluyeron todos los escolares del rango de edad establecido cuyos padres firmaron el consentimiento informado y además dieron su asentimiento, excluyéndose del estudio a quienes no lo hicieron y se encontraban bajo tratamientos de Ortodoncia.Se utilizó una entrevista estructurada para la aplicación del Child-OIDP,el examen clínico y la escala estética del INTO, luego de la respectiva calibración y la prueba piloto. Se obtuvo la aprobación del Comité Institucional de Ética de la Universidad Peruana Cayetano Heredia, así como las autorizaciones respectivas de las autoridades educativas. El proceso de recolección de datos se cumplió en tres etapas, primero se obtuvo la información de los datos generales, luego se realizó la entrevista (CS Child-OIDP) y finalmente el examen clínico (INTO CSD) y la selección de una fotografía. (INTO CE) ; el índice se aplicó a través de entrevistas individuales a los niños, preguntando si en los últimos tres meses han tenido problemas respecto de posición de los dientes, espacios, tamaño y forma y deformidades craneofacialesy si esos problemas han causado dificultad en los desempeños tales como: comer, hablar, limpiarse la boca, dormir, emoción, sonreír, estudiar y socializar; posteriormente se interrogó acerca de la severidad del impacto en el desempeño en una escala de 1 a 3 así como la frecuencia del impacto en el desempeño igualmente en una escala de 1 a 3 . Para determinar la intensidad del impacto de las condiciones orales relacionadas a maloclusiones se utilizó la clasificación sugerida por Gherunpong y colaboradores. La extensión del impacto de las condiciones relacionadas a maloclusiones se refiere al número de desempeños diarios afectados. En la siguiente etapa se evaluó a los niños a través del INTO, para determinar el CE-INTO se presentaron 10 fotografías intraorales ordenadas desde la 1 hasta la 10 en dos columnas, donde la número 1 estaba catalogada como la mejor y la 10 la peor; se le pidió al niño que busque la foto de acuerdo a la percepción de su estética dental, si la foto elegida se encontraba entre la 1 y la 4 , se considera sin necesidad de tratamiento; entre la 5 y 7 , con necesidad moderada de tratamiento; y entre la 8 y 10, con necesidad urgente de tratamiento. Para determinar el componente de salud dental del INTO serealizó un examen clínico a los niños, y se observó las características de los siguientes rasgos oclusales: overjet, overbite, mordida abierta, mordida cruzada, erupción impedida, defectos de labio leporino y paladar hendido, anomalías craneofaciales, erupción impedida e hipodoncia. , la medición se la hizo con una sonda periodontal y un tope para poder verificar la misma en un dentímetro; el rasgo oclusal más alterado se utilizó para categorizar la necesidad de tratamiento de ortodoncia desde sin necesidad de tratamiento (grado $1 \mathrm{y}$ 2), necesidad moderada (grado 3) y con necesidad de tratamiento (grado 4 y 5). Elplan de análisis consistió en la obtención de frecuencias absolutas y relativas de las variables cualitativas, y promedios y desviación estándar de la variable cuantitativa. El análisis bivariado se llevó a cabo mediante la Prueba de Chi cuadrado, Chi cuadrado corregido por Yates, Prueba de U de Mann Whitney, y Prueba de Kruskall Wallis (comprobación de distribución z mediante la Prueba Kolmogorov Smirnov). Se utilizaron los programas Excel y SPSS v. 23. El estudio contó con un nivel de confianza de $95 \%$ y un $\mathrm{p}<0,05$.

\section{RESULTADOS}

Se evaluaron 170 niños de 11 y 12 años de edad que cumplieron con los criterios de selección todos pertenecientes al área urbana de la ciudad; la muestra presentó una distribución de 91 niñas y 79 niños, de ellos 77 fueron de 11 años y 93 de 12 años de edad. 
Tabla 1. Prevalencia de las condiciones orales atribuidas a maloclusiones percibidas como problemas por los escolares de 11-12 años de las escuelas privadas Providencia y César Cordero Moscoso de la ciudad de Azogues-Ecuador 2015.

\begin{tabular}{lcccc}
\hline \multicolumn{1}{c}{ FRECUENCIA } & \multicolumn{2}{c}{ Presente } & \multicolumn{2}{c}{ Ausente } \\
& $\mathbf{n}$ & $\mathbf{\%}$ & $\mathbf{n}$ & $\mathbf{\%}$ \\
\hline Separados & 69 & 40,59 & 101 & 59,41 \\
Forma & 49 & 28,82 & 121 & 71,18 \\
Posición & 108 & 63,53 & 62 & 36,47 \\
\hline
\end{tabular}

n: Frecuencia absoluta.

$\%$ : Frecuencia relativa.

Tabla 2. Prevalencia de las condiciones orales atribuidas a maloclusiones percibidas como problemas por los escolares de 11-12 años según género de las escuelas privadas Providencia y César Cordero Moscoso de la ciudad de Azogues-Ecuador 2015

\begin{tabular}{lccccc}
\hline \multirow{2}{*}{ FRECUENCIA } & \multicolumn{2}{c}{ GÉNERO } \\
& $\mathbf{2}$ Masculino & \multicolumn{2}{c}{ Femenino } & \\
& $\mathbf{n}$ & $\mathbf{\%}$ & $\mathbf{n}$ & $\mathbf{\%}$ & $\mathbf{p}^{*}$ \\
\hline Separados & 34 & 20,00 & 35 & 20,59 & 0,54 \\
Forma & 22 & 12,94 & 27 & 15,88 & 0,79 \\
Posición & 46 & 27,06 & 62 & 36,47 & 0,18 \\
\hline
\end{tabular}

n: Frecuencia absoluta.

\%: Frecuencia relativa.

p: Significancia estadística.

*: Prueba de Chi-cuadrado.

Tabla 3. Comparación del impacto de las maloclusiones sobre los desempeños diarios en los escolares de 11 y 12 años según género, de las escuelas Providencia y César Cordero Moscoso, Azogues-Ecuador 2015

\begin{tabular}{lccccc}
\hline & \multicolumn{5}{c}{ GÉNERO } \\
PREVALENCIA & \multicolumn{2}{c}{ Masculino } & \multicolumn{2}{c}{ Femenino } & p \\
& $\mathbf{n}$ & $\mathbf{\%}$ & $\mathbf{n}$ & $\mathbf{\%}$ & $0.72^{*}$ \\
Comer & 36 & 21,18 & 39 & 22,94 & $0.89^{*}$ \\
Hablar & 11 & 6,47 & 12 & 7,06 & $0.29^{*}$ \\
Limpiar & 17 & 10,00 & 26 & 15,29 & $0.45^{\circ}$ \\
Descansar & 2 & 1,18 & 5 & 2,94 & $0.96^{*}$ \\
Ánimo & 8 & 4,71 & 9 & 5,29 & $0.76^{*}$ \\
Sonreír & 26 & 15,29 & 32 & 18,82 & $1.00^{\circ}$ \\
Tarea & 1 & 0,59 & 2 & 1,18 & $0.54^{*}$ \\
Amigo & 14 & 8,24 & 13 & 7,65 & \\
\hline
\end{tabular}

n: Frecuencia absoluta.

$\%$ : Frecuencia relativa.

p: Significancia estadística.

*: Prueba de Chi-cuadrado.

o: Prueba de Chi-cuadrado corregido por Yates. 
Tabla 4. Intensidad del impacto de las condiciones orales atribuidas a maloclusiones sobre los desempeños diarios en los escolares de 11 y 12 años, según sexo, de las escuelas Providencia y César Cordero Moscoso.

\begin{tabular}{|c|c|c|c|c|c|c|c|c|c|}
\hline Intensidad (\%) & & & & & Actividades & Diarias & & & \\
\hline & 占 & 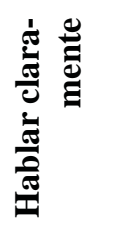 & 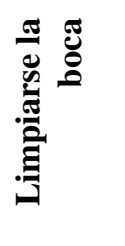 & 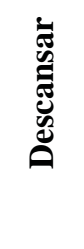 & 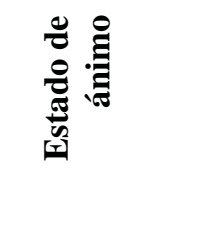 & 党 & 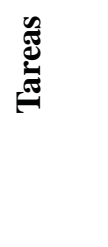 & 总 & 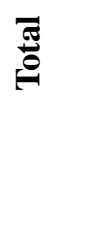 \\
\hline & (\%) & (\%) & (\%) & (\%) & $(\%)$ & (\%) & $(\%)$ & $(\%)$ & (\%) \\
\hline FEMENINO & 8,70 & 7,61 & 10,87 & 2,17 & 4,35 & 8,70 & 2,17 & 7,61 & 46,74 \\
\hline \multicolumn{10}{|l|}{ Muy Роса } \\
\hline Poca & 25,00 & 3,26 & 17,39 & 3,26 & 6,52 & 16,30 & 1,09 & 7,61 & 19,57 \\
\hline Regular & 16,30 & 7,61 & 11,96 & 5,43 & 5,43 & 14,13 & 2,17 & 8,70 & 19,57 \\
\hline Severa & 9,78 & 1,09 & 6,52 & 1,09 & 1,09 & 10,87 & 0 & 2,17 & 2,17 \\
\hline Muy Severa & 6,52 & 3,26 & 2,17 & 0 & 3,26 & 3,26 & 1,09 & 2,17 & 0 \\
\hline \multicolumn{10}{|l|}{ MASCULINO } \\
\hline Muy Роса & 14,1 & 5,13 & 5,13 & 3,85 & 5,13 & 7,69 & 3,85 & 3,85 & 42,31 \\
\hline Poca & 10,26 & 3,85 & 15,38 & 1,28 & 5,13 & 12,82 & 0 & 6,41 & 29,49 \\
\hline Regular & 23,08 & 5,13 & 6,41 & 1,28 & 2,56 & 17,95 & 1,28 & 10,26 & 15,38 \\
\hline Severa & 11,54 & 6,41 & 5,13 & 1,28 & 3,85 & 7,69 & 0 & 2,56 & 0 \\
\hline Muy Severa & 5,13 & 0 & 0 & 0 & 0 & 3,85 & 0 & 1,28 & 0 \\
\hline Valor p & 0,89 & 0,8 & 0,007 & 0,23 & 0,42 & 0,71 & 0,4 & 0,76 & \\
\hline
\end{tabular}

Prueba Chi Cuadrado

* Prueba Exacta de Fisher 
Tabla 5. Comparación de la Extensión del impacto de las condiciones orales atribuidas a maloclusiones sobre los desempeños diarios en los escolares de 11 y 12 años, según género, de las escuelas Providencia y César Cordero Moscoso, Azogues-Ecuador 2015.

\begin{tabular}{lllllll}
\hline \multirow{2}{*}{ EXTENSIÓN } & \multicolumn{5}{c}{ GÉNERO } \\
& \multicolumn{2}{c}{ Masculino } & \multicolumn{2}{c}{ Femenino } & \\
& $\mathbf{X}$ & $\mathbf{D E}$ & $\mathbf{X}$ & $\mathbf{D E}$ & $\mathbf{p}^{*}$ \\
\hline Comer & 0,65 & 0,48 & & 0,66 & 0,48 & 0,85 \\
Hablar & 0,29 & 0,46 & 0,33 & 0,47 & 0,59 \\
Limpiar & 0,33 & 0,47 & 0,48 & 0,50 & 0,04 \\
Descansar & 0,09 & 0,29 & 0,12 & 0,33 & 0,50 \\
Ánimo & 0,16 & 0,37 & 0,21 & 0,41 & 0,46 \\
Sonreír & 0,49 & 0,50 & 0,54 & 0,50 & 0,56 \\
Tarea & 0,05 & 0,22 & 0,07 & 0,25 & 0,67 \\
Amigo & 0,24 & 0,43 & 0,30 & 0,46 & 0,41 \\
\hline
\end{tabular}

X: Promedio.

DE: Desviación estándar.

p: Significancia estadística.

*: Prueba de U de Mann Whitney.

Tabla 6. Severidad del impacto de las condiciones orales atribuidas a maloclusiones sobre los desempeños diarios en los escolares de 11 y 12 años, de las escuelas Providencia y César Cordero Moscoso, Azogues-Ecuador 2015.

\begin{tabular}{lcccc}
\hline \multicolumn{1}{c}{ Actividades diarias } & Media & D.E. & $\begin{array}{c}\text { Valor } \\
\text { Mínimo }\end{array}$ & $\begin{array}{c}\text { Valor } \\
\text { Máximo }\end{array}$ \\
\hline Comer Alimentos & 2,39 & 2,582 & 0 & 9 \\
Hablar Claramente & 0,76 & 1,837 & 0 & 9 \\
Limpiarse la Boca & 1,23 & 1,925 & 0 & 9 \\
Descansar & 0,26 & 0,939 & 0 & 6 \\
Buen Estado de Animo & 0,63 & 1,685 & 0 & 9 \\
Sonreír & 1,85 & 2,402 & 0 & 9 \\
Realizar labores escolares & 0,16 & 0,866 & 0 & 9 \\
Contactarse con otras personas & 0,86 & 1,820 & 0 & 9 \\
Total & 8,16 & 7,704 & 0 & 39 \\
\hline
\end{tabular}


Tabla 7. Necesidad de tratamiento ortodóntico según el componente estético (CE) y el componente de salud dental (CSD) del INTO en escolares de 11 a 12 años de los Colegios Providencia y Cesar Cordero Moscoso, Azogues-Ecuador 2015.

\begin{tabular}{llcc}
\hline \multicolumn{1}{c}{ NECESIDAD DE TRATAMIENTO ORTODÓNTICO } & $\mathbf{n}$ & $\mathbf{\%}$ \\
\hline Componente clínico & & 17 & 10,00 \\
& Sin necesidad de tratamiento & 99 & 58,24 \\
& Leve sin necesidad de tratamiento & 53 & 31,18 \\
& Moderada límite de necesidad de tratamiento & 1 & 0,59 \\
& Grave necesidad de tratamiento & 0 & 0,00 \\
& extrema necesidad de tratamiento & 170 & 100,00 \\
& total & & \\
& & 155 & 91,18 \\
& Sin necesidad de tratamiento & 8 & 4,71 \\
& Necesidad moderada de tratamiento & 7 & 4,12 \\
& Necesidad de tratamiento & 170 & 100,00 \\
\hline
\end{tabular}

n: Frecuencia absoluta.

$\%$ : Frecuencia relativa.

Tabla 8. Necesidad de tratamiento ortodóntico según el componente estético (CE) y el componente de salud dental (CSD) del INTO en escolares de 11 a 12 años, según género de los Colegios Providencia y Cesar Cordero Moscoso, Azogues-Ecuador 2015

\begin{tabular}{|c|c|c|c|c|c|}
\hline \multirow{3}{*}{$\begin{array}{l}\text { NECESIDAD DE TRATAMIENTO ORTODÓNTICO } \\
\text { Componente clínico }\end{array}$} & \multicolumn{5}{|c|}{ GÉNERO } \\
\hline & \multicolumn{2}{|c|}{ Masculino } & \multicolumn{2}{|c|}{ Femenino } & \multirow[b]{2}{*}{$\mathbf{p}^{*}$} \\
\hline & $\mathbf{n}$ & $\%$ & $\mathbf{n}$ & $\%$ & \\
\hline Sin necesidad de tratamiento & 7 & 4,12 & 10 & 5,88 & \\
\hline Leve sin necesidad de tratamiento & 38 & 22,35 & 61 & 35,88 & \\
\hline Moderada límite de necesidad de tratamiento & 34 & 20,00 & 19 & 11,18 & 0,02 \\
\hline Grave necesidad de tratamiento & 0 & 0,00 & 1 & 0,59 & \\
\hline Extrema necesidad de tratamiento & 0 & 0,00 & 0 & 0,00 & \\
\hline \multicolumn{6}{|l|}{ Componente estético } \\
\hline Sin necesidad de tratamiento & 71 & 41,76 & 84 & 49,41 & \\
\hline Necesidad moderada de tratamiento & 4 & 2,35 & 4 & 2,35 & 0,54 \\
\hline Necesidad de tratamiento & 4 & 2,35 & 3 & 1,76 & \\
\hline
\end{tabular}

n: Frecuencia absoluta.

\%: Frecuencia relativa.

p: Significancia estadística.

*: Prueba de Chi-cuadrado corregido por Yates. 
Tabla 9. Prevalencia del impacto de las condiciones orales atribuidas a maloclusiones sobre los desempeños diarios según componente clínico del INTO en escolares de 11 y 12 años de las escuelas Providencia y Cesar Cordero Moscoso, Azogues-Ecuador 2016

\section{NECESIDAD DE TRATAMIENTO ORTODÓNTICO \\ Componente clínico}

\begin{tabular}{|c|c|c|c|c|c|c|c|c|c|}
\hline \multirow[t]{2}{*}{ Desempeños } & \multicolumn{2}{|c|}{ 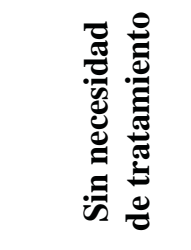 } & \multicolumn{2}{|c|}{ 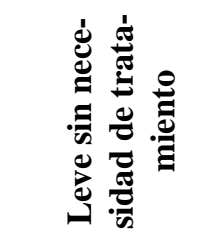 } & \multicolumn{2}{|c|}{ 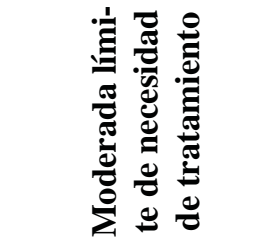 } & \multicolumn{2}{|c|}{ 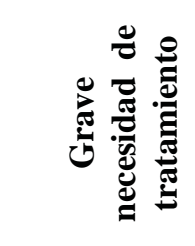 } & \multirow[t]{2}{*}{$\mathbf{p}^{*}$} \\
\hline & $\mathbf{n}$ & $\%$ & $\mathbf{N}$ & $\%$ & $\mathbf{n}$ & $\%$ & $\mathbf{n}$ & $\%$ & \\
\hline Comer & 5 & 2.94 & 46 & 27.06 & 23 & 13.53 & 1 & 0.59 & 0.42 \\
\hline Hablar & 1 & 0.59 & 13 & 7.65 & 9 & 5.29 & 0 & 0.00 & 0.30 \\
\hline Limpiar & 2 & 1.18 & 26 & 15.29 & 15 & 8.82 & 0 & 0.00 & 0.34 \\
\hline Descansar & 0 & 0.00 & 2 & 1.18 & 5 & 2.94 & 0 & 0.00 & 0.03 \\
\hline Ánimo & 2 & 1.18 & 10 & 5.88 & 4 & 2.35 & 1 & 0.59 & 0.94 \\
\hline Sonreír & 4 & 2.35 & 29 & 17.06 & 24 & 14.12 & 1 & 0.59 & 0.02 \\
\hline Tarea & 0 & 0.00 & 1 & 0.59 & 2 & 1.18 & 0 & 0.00 & 0.21 \\
\hline Amigo & 2 & 1.18 & 14 & 8.24 & 11 & 6.47 & 0 & 0.00 & 0.32 \\
\hline
\end{tabular}

Tabla 10. Impacto de las condiciones orales atribuidas a maloclusiones en escolares de 11 a 12 años de las escuelas Providencia y César Cordero Moscoso, según género y necesidad de tratamiento ortodóntico. Azogues-Ecuador 2015

\begin{tabular}{|c|c|c|c|c|c|c|}
\hline & & \multicolumn{2}{|c|}{ Sin impacto } & \multicolumn{2}{|c|}{ Con impacto } & \multirow{3}{*}{$\mathbf{p}^{*}$} \\
\hline & & $\mathbf{n}$ & $\%$ & n & $\%$ & \\
\hline & Total & 4 & 2,02 & 166 & 97,98 & \\
\hline \multirow[t]{2}{*}{ Sexo } & Hombre & 2 & 1,18 & 77 & 45,29 & 1 \\
\hline & Mujer & 2 & 1,18 & 89 & 52,35 & \\
\hline \multirow[t]{5}{*}{$\begin{array}{l}\text { CSD } \\
\text { IOTN }\end{array}$} & Sin necesidad de tratamiento & 1 & ,59 & 16 & 9,41 & 0,47 \\
\hline & Leve sin necesidad de tratamiento & 2 & 1,18 & 97 & 57,06 & \\
\hline & Moderada límite de necesidad de tratamiento & 1 &, 59 & 52 & 30,59 & \\
\hline & Grave necesidad de tratamiento & 0 & 0,00 & 1 & ,59 & \\
\hline & Extrema necesidad de tratamiento & 0 & 0,00 & 0 & 0,00 & \\
\hline \multirow[t]{3}{*}{$\begin{array}{l}\text { CE } \\
\text { IOTN }\end{array}$} & Sin necesidad de tratamiento & 4 & 2,35 & 151 & 88,82 & 0,55 \\
\hline & Necesidad moderada de tratamiento & 0 & 0,00 & 8 & 4,71 & \\
\hline & Necesidad de tratamiento & 0 & 0,00 & 7 & 4,12 & \\
\hline
\end{tabular}

$\%$ : Frecuencia relativa.

p: Significancia estadística.

*: Prueba de Chi-cuadrado corregido por Yates

Fisher 
En cuanto a la condición más prevalente fue posición de los dientes (63,5\%) y de igual forma según género con un porcentaje mayor para el femenino (36,47\%) sin existir diferencia estadísticamente significativa (tabla 1 y tabla 2 ).

Al comparar según género los desempeños más prevalentes fueron comer y sonreír en el sexo masculino (21,18\% y $15,29 \%$ respectivamente) y para el femenino las mismas actividades en el mismo orden de prevalencia (22,94\% y 18,82\% respectivamente), no se encontraron diferencias estadísticamente significativas (tabla 3).

Respecto de la intensidad del impacto sobre los desempeños diarios según género , en el caso del femenino, los escolares manifestaron que el desempeño con intensidad severa más afectado fue sonreír (10,87\%) e intensidad muy severa comer (6,52\%); en cuanto a sexo masculino los escolares manifestaron, el desempeño con intensidad severa y muy severa más afectado fue comer (11,54\%, 5,13\%); no se encontró diferencia estadísticamente significativa (tabla 4).

En cuanto a la extensión del impacto de maloclusiones sobre los desempeños diarios según género, en el caso de masculino el desempeño comer tuvo el promedio más alto $0,65 \pm 0.48$ seguido de sonreír $0,49 \pm$ 0,5 ; y en el sexo femenino los resultados fueron similares, comer $0,66 \pm 0.48$ y sonreír $0,54 \pm 0,50$ no se encontraron diferencias significativas (tabla 5). La severidad del impacto sobre los desempeños diarios tuvo el mayor valor promedio en el desempeño comer un valor promedio de 2,39 seguido de sonreír 1,85 con un rango entre 0 y 9 (tabla 6).

Respecto de la Necesidad de Tratamiento ortodóntico percibida por los escolares según el Componente Estético del INTO se encontró que un 91,18\% de los escolares no requerían tratamiento de ortodoncia desde su autopercepción, y solo el 4,12\% requerían tratamiento; según el componente clínico, se encontró que 10\% no necesitaban tratamiento, el 58,24\% tenían una necesidad leve, un 31,18\% necesidad moderada o límite de tratamiento y un 0,59 \% con necesidad urgente de tratamiento (tabla 7).

La necesidad de Tratamiento por género, determinó según el componente clínico que lo prevalente en ambos casos fue la condición leve sin necesidad de tra- tamiento 22,35\% para el masculino y 35,88\% para el femenino, encontrándose diferencia estadísticamente significativa $\mathrm{p}=0,02$; según el componente estético en ambos sexos se observó que la condición sin necesidad de tratamiento fue la más prevalente $41,76 \%$ para el masculino 49,41\% para el femenino, no se observó significancia estadística (tabla 8).

En cuanto a la prevalencia del impacto de las condiciones orales atribuidas a maloclusiones sobre los desempeños diarios según necesidad de tratamiento, se observó que los desempeños comer y sonreír fueron los más afectados (27,06\% y 17,06\% respectivamente) con una necesidad leve de tratamiento solamente en el componente clínico se encontró diferencia estadísticamente significativa en el desempeño sonreír p=0,02 (tabla 9).

El impacto de las maloclusiones sobre la calidad de vida y necesidad de tratamiento ortodóntico concluye en determinar que se presentó especialmente en los niños con necesidad leve y sin necesidad de tratamiento de acuerdo a las categorías clínica y estética respectivamente; sin existir diferencia estadísticamente significativa (tabla 10).

\section{DISCUSIÓN}

En este estudio se consideró a los niños de 11 y 12 años en razón de que por un lado el índice Child-OIDP originalmente se lo confeccionó y validó con este grupo de edad (8); y además la organización Mundial de la Salud recomienda este rango para efectuar la vigilancia epidemiológica de las enfermedades bucales, y poder realizar comparaciones internacionales (9). De otro lado el INTO igualmente se validó en 1989 para su utilización en escolares de este grupo etario, y lo han respaldado varios estudios realizados posteriormente a su promulgación $(6,10,11)$.

El tamaño de la muestra correspondió a 170 escolares de 11 y 12 años, de los cuales fueron 91 niñas y 79 niños que cumplieron con los criterios de selección, específicamente lo pertinente a no haber recibido o estar con tratamiento ortodóntico u ortopédico, criterios similares lo aplicaron los estudios de Ricse y Manccini en Lima y Souames en Francia $(3,6,12)$. Algunos estudios realizados en zonas urbanas y urbano marginales han utilizado el Chid OIDP en base a 17 condiciones orales generales que podrían ser 
percibidas como problemas, en este estudio se aplicó el mismo índice pero basado en condiciones orales atribuidas a maloclusiones, y de esa forma detectar aspectos relacionados a una enfermedad o situación clínica, para que el escolar pueda discriminar mejor los problemas e impactos relacionados solo a maloclusiones. $(6,11,13-15)$.

Respecto de las condiciones orales atribuidas a maloclusiones autopercibidas como problemas, posición de los dientes fue la más prevalente (63,5\%), estos resultados coinciden con algunos estudios como el de Bianco en el año 2006 en Italia (16), Bernabé en Zapallal Perú (11) y Ricse en el año 2011 en el distrito de San Juan de Lurigancho Lima (6), con la diferencia que en ese estudio la mayor prevalencia la tuvo la de dientes separados con 53\% seguida de posición de los dientes $21 \%$. De otro lado este resultado difiere con otros como el de Gherunpong et al., cuya condición más afectada fue diente sensible 27,9\% (14), Del Castillo en el año 2010 en Piura cuya condición más prevalente fue dolor de muela 58\% (17), al igual que Ingunza en su estudio de zonas urbano-marginales de Lima (18); la diferencia podría radicar en que son estudios realizados en zonas urbano marginales y rurales a diferencia de este estudio que se realizó en zona urbana.

Respecto de la prevalencia general de desempeños afectados en este estudio fue del $87,6 \%$, similar a los estudios realizados por Del Castillo 88,7\% (17), Gherunpong 89,8\% (14), Paredes en Lima 100\% (9), Castro 80,7\% en la validación del Índice en Brasil (19), diferentes a los reportados por Bianco 66,8\% en Italia (16), Ricse 33,3\% (6), Bernabé 15,5\% (11),y Bernabé en Baurú 24,6\% (15).

Los desempeños más afectados en este estudio corresponden a comer y sonreír (65,3\% y 51,8\%), resaltando la coincidencia del desempeño sonreír como uno de los más afectados en varios estudios como el de Ricse (30,4\%), Bianco (29,9\%), Castro, Paredes y Díaz, Del Castillo y Bernabé $(6,9,15-17,19)$.

El desempeño sonreír ha demostrado en este estudio ser uno de los más afectados, igual sucede en otros estudios como los de Ricse (6), Bernabé (11) y Gherunpong (14); en otros estudios como los de Bianco (16), Del Castillo (17) y Paredes (9) se reportan otros desempeños como los más severamente afectados.
Respecto de la necesidad de tratamiento ortodóntico obtenida clínicamente en este estudio determinó que un $31,18 \%$ de los escolares presentaba necesidad moderada de tratamiento, porcentaje que se lo considera importante, debido a que en esta edad se suscitan varios acontecimientos de discrepancias dentoalveolares, y de otro lado observar varios problemas de mal posición en este grupo escolar se atribuye a que varios de los escolares aún no han podido acceder a la consulta privada, pues no existe por parte del estado una atención especializada para problemas ortodónticos en los diferentes niveles de atención del Ministerio de Salud Pública del Ecuador, los resultados obtenidos con esta categoría clínica del INTO en este estudio coinciden con algunos otros como los de Bernabé 34,9\% (20), Brook y Shaw 33\% (10), y ligeramente superior a lo encontrado por Manccini 29,1\% (3), Souames $29,5 \%$ (12), Nguyen $21 \%$ (21) y Ricse $(24,6 \%)(6)$, y difieren con porcentajes menores, de los estudios de Pardo 19,52\% (23).

En cuanto al componente estético el mayor porcentaje estuvo en los escolares sin necesidad de tratamiento (91,18\%), resultado mayor en relación a los estudios de Ricse con 78,2\% (6), Bernabé 87,2\% (20), Manccini $88,6 \%$ (3), Souames 75,4\% (12), Pardo 83,3\% (22), Kumar 73,6\% (23), Brook y Shaw (10), cuya prevalencia según este componente fue relativamente baja (2\%). Cabe el análisis en el sentido que estos resultados aunque no presentan coincidencias exactas en sus porcentajes reflejan en todos ellos mayoritariamente la percepción del escolar desde una perspectiva de innecesario tratamiento ortodóntico, posiblemente debido al número de fotografías que se agrupan en cada valor de la escala (10) , y de otro lado influiría también el hecho de que una condición clínica manifiesta como problema, para el escolar no lo sea, ello permite inferir una cierta apreciación subjetiva en los escolares quienes no ven según su autopercepción a una malposicion dentaria como algo que afecte o preocupe a su estética.

Al comparar los componentes del INTO según sexo, el componente de Salud Dental presentó una diferencia estadísticamente significativa (p 0,02), a diferencia del componente estético en donde no hubo diferencia estadísticamente significativa ( 0,54$)$; estos resultados mostraron similitud con Manccini, Perillo y diferente al de Ricse, y Souames; el estudio de Nguyen en población Vietnamita encontró diferencia estadísticamente significativa pero en el Componente Estético (21). 
Es importante destacar que no existe una correspondencia entre los dos componentes del INTO así lo demuestran los estudios de Ricse et al., e Ichiki en el Perú $(6,24)$, en este mismo sentido Bernabé y Flores concluyen indicando que la necesidad normativa de tratamiento de ortodoncia no fue acompañada de un nivel similar de necesidad de tratamiento percibido por los estudiantes (20).

Estos aspectos coincidentes permiten hacer una reflexión desde el punto de vista de la autopercepción del niño de esa edad (11 y 12 años), que minimiza o desestima en realidad un problema estético atribuible a sus dientes, surge por tanto un aspecto "contradictorio" pues si bien por un lado manifiestan la posición dentaria como la condición o problema que más afecta sus desempeños diarios especialmente el comer y sonreír, sin embargo en el momento de aplicar el INTO según el Componente Estético consideran poco relevante la necesidad de tratamiento; la explicación posiblemente se atribuiría a situaciones que colocan al autoconcepto como una dimensión que no se diferencia internamente sino hasta entrada la adolescencia(25); es decir la contraposición de los resultados tendría de un lado una explicación desde el punto de vista del desarrollo psicológico-biológico y afectivo, y de otro lado amerita colocar en el plano de la discusión la posibilidad de ampliar los estudios con este grupo de edad para resultados todavía más concluyentes.

En cuanto al impacto de las condiciones orales atribuidas a maloclusiones sobre los desempeños diarios considerando la necesidad de tratamiento, se observó que los desempeños comer y sonreír fueron los más afectados en las dos categorías CSD y CE, con significancia estadística solo en el desempeño sonreír (p 0,02), aspecto similar al encontrado en estudios como Ricse (6) y Bernabé $(11,15)$

El impacto de las condiciones orales sobre la calidad de vida según necesidad de tratamiento reveló que el mayor impacto estuvo en los niños con Necesidad Leve y Sin Necesidad de acuerdo a las categorías clínica y estética respectivamente; sin encontrarse diferencia estadísticamente significativa, aspecto similar al encontrado en el estudio de Bernabé (11) y diferente al encontrado por Ricse (6) y Bernabé $(13,26)$.

Lo reportado en este estudio serviría para tener un claro panorama respecto de cómo los escolares pueden mejorar su bienestar con menos impacto en sus des- empeños diarios (hablar, comer, sonreír, etc.) tratando de solucionar los problemas bucales atribuidos a maloclusión como posición, espacios, forma o tamaño de los dientes, etc. que afectan su calidad de vida, y que la necesidad de tratamiento va de la mano con esa problemática; en tal virtud esclarecerlo permitirá solucionar los aspectos concomitantes a un problema de malposicion dentaria o dentofacial, que repercutirá indiscutiblemente en su diario vivir, valorando lo importante que sería ver a un adolescente desarrollarse en una etapa tan particular o compleja en las mejores condiciones posibles.

\section{CONCLUSIONES}

El impacto de las condiciones orales atribuidas a maloclusiones y la necesidad de tratamiento ortodóntico en los escolares de 11 y 12 años en dos escuelas privadas de la ciudad de Azogues permitió considerar que la percepción fue de un gran impacto en los niños con necesidad leve y sin necesidad de tratamiento según componentes clínicos y estético del IOTN respectivamente; considerando sin embargo que la necesidad normativa de tratamiento de ortodoncia no fue acompañada de un nivel similar de necesidad de tratamiento percibido por los estudiantes. Tanto la autopercepción del problema ortodóntico, o que sea el profesional quien lo determine, permite abrir al niño y sus padres todo un campo amplio de problemas que pueden ser solucionados por el especialista (Ortodoncista), a fin de conseguir una mejor condición de su calidad de vida, o que estos estudios, referenciados en varios países del mundo, puedan plantear la posibilidad de que los establecimientos públicos de salud puedan ofrecer alternativas de tratamiento ortodóntico en los niveles de atención adecuados para ello.

\section{Correpondencia:}

Cristina Crespo Crespo

Correo electrónico: michi_cres@yahoo.es

\section{REFERENCIAS BIBLIOGRÁFICAS}

1. Puerta I, Díaz S. Repercusiones de la salud bucal sobre calidad de vida por ciclo vital individual. Acta Odontológica Colombiana. 2017; 7 (2): 49-64.

2. Isper AJ, Pantaleão dos Santos MR, Gonçalves PE. Prevalencia de maloclusión en la dentición primaria en el municipio de Cáceres Brasil. Rev Cubana Estomatol. 2007; 45(1):91-95 
3. Manccini L.M. Necesidad de tratamiento ortodóntico según el índice de necesidad de tratamiento ortodóntico en escolares de 12 a 13 años. Tesis para obtener título profesional de Cirujano Dentista. Lima, Perú: Universidad Nacional Mayor de San Marcos; 2011.

4. Preciado M. Relación entre tipo de maloclusión, patrón facial y autoestima en sujetos de 14 a 17 años de edad de un colegio en Trujillo. Perú. 2013. Rev Simiykita. 2015; 1(2):64-71.

5. Misrachi C, Espinoza I. Utilidad de las mediciones de la calidad de vida relacionada con la salud. Rev Dent Chile. 2005; 96 (2): 28-35.

6. Ricse E. Impacto de las condiciones orales atribuidas a maloclusiones sobre la calidad de vida y la necesidad de tratamiento ortodóntico en escolares de 11 a 12 años de un colegio de un distrito urbano, San Juan de Lurigancho. Tesis de Maestría en Estomatología. Lima, Perú: Universidad Peruana Cayetano Heredia; 2011.

7. Paredes R. El impacto de las condiciones orales sobre la calidad de vida en escolares del distrito de Villa El Salvador. Tesis Maestria en Estomatología Lima, Perú: Universidad Peruana Cayetano Heredia; 2013.

8. Gherunpong S, Tsakos G, Sheiham A. A socio-dental approach to assessing children's orthodontic needs. Eur J Orthod. 2006; 28: 393-9.

9. Paredes E, Díaz M. Impacto de las condiciones orales sobre la calidad de vida en escolares del Distrito de San Juan de Miraflores. Rev Estomatol Herediana. 2014; 24(3): 171-175.

10. Brook P, Shaw W. The development of an index of orthodontic treatment priority. Eur J Orthod. 1989;11(3):309-20.

11. Bernabé E, Flores C, Sheiham A. Prevalence, intensity and extent of Oral Impacts on Daily Performances associated with self-perceived Malocclusion in 11-12-year-old children. BMC Oral Health. 2007; 7: 6.

12. Souames M, Bassigny F, Zenati N, Riordan PJ, BoyLefevre ML. Orthodontic treatment need in French schoolchildren: an epidemiological study using the Index of Orthodontic Treatment Need. Eur J Orthod. 2006;28(6):605-9.

13. Bernabé E, Sheiham A, Tsakos G, de Oliveira C. The impact of orthodontic treatment on the quality of life in adolescents: a case - control study. Eur J Orthod. 2008; 30(5):515-20. doi: 10.1093/ejo/cjn026

14. Gherunpong S, Tsakos G, Sheiham A. A socio-dental approach to assessing children's orthodontic needs. Eur J Orthod. 2006; 28(4):393-9.

15. Bernabé E, Tsakos G, Messias de Oliveira C, Sheiham A. Impacts on daily performances attributed to malocclusions using the condition-specific feature of the Oral Impacts on Daily Performances Index. Angle Orthod. 2008;78(2):241-7. doi: 10.2319/030307-111.1.

16. Bianco A, Fortunato L, Giuseppe A, Pavia M.
Prevalence and determinants of oral impacts on daily performance: results from a survey among School children in Italy. E J of Public Health. 2009; 20(5): 595-600.

17. Del Castillo-López C, Evangelista-Alva A, SánchezBorjas P. Impacto de las condiciones bucales sobre la calidad de vida en escolares de ámbito rural Piura, Perú Rev Estomatol Herediana. 2014;24(4):220-228.

18. Marcelo-Ingunza J, Del Castillo-López C, Evangelista-Alva A, Sánchez-Borjas P. Calidad de vida relacionada a la salud bucal en escolares de ámbito urbano-marginal. Rev Estomatol Herediana. 2015;25(3):194-204.

19. Castro R, Cortes M, Leão A, et al.Child-OIDP index in Brazil: Cross-cultural adaptation and validation. Health Qual Life Outcomes. 2008; 6: 68.

20. Bernabé E, Flores C. Normative and self-perceived orthodontic treatment need of a Peruvian university population. Head Face Med. 2006; 3: 2-22.

21. Nguyen S, Nguyen M, Sagg M, Jagomagi T. The need for orthodontic treatment among Vietnamese school children and young adults. Int J Dent. 2014; 2014: 132301. doi: 10.1155/2014/132301

22. Pardo K. Índice de necesidad de tratamiento ortodóncico en escolares del nivel de secundaria de la i.e. no 154 “Carlos Noriega Jiménez. Tesis para optar titulo de Cirujano Dentista. Lima, Perú: Universidad San Martin De Porres; 2012.

23. Kumar P, Londhe B, Kotwal A, Mitra R. Prevalence of malocclusion and orthodontic treatment need in school children an epidemiological study. MJAFI. 2013; 6: 369-37.

24. Ichiki ME. Asociación entre la necesidad de tratamiento ortodóntico evaluada mediante el IOTN y el DAI en adultos Nissei del Policlínico Peruano Japonés. Tesis Maestria en Estomatología. Lima, Perú: Universidad Peruana Cayetano Heredia; 2003.

25. Fernández A, Goñi E. El autoconcepto infantil: una revisión necesaria. International Journal of Developmental and Educational Psychology. 2008;2(1): 13-22.

26. Bernabé E, Sheiham A, Tsakos G, de Oliveira C. The impact of orthodontic treatment on the quality of life in adolescents: a case - control study. Eur J Orthod. 2008; 30(5):515-20. doi: 10.1093/ejo/cjn026

Recibido: 25-03-2017

Aceptado: 04-06-2017 